\title{
Combined Toxicity of Cannabidiol Oil with Three Bio-Pesticides against Adults of Sitophilus Zeamais, Rhyzopertha Dominica, Prostephanus Truncatus and Trogoderma Granarium
}

\author{
Spiridon Mantzoukas ${ }^{1, * \mathbb{D}}$, Nikolaos Kalyvas ${ }^{1}$, Aristeidis Ntoukas ${ }^{1}$, Ioannis Lagogiannis ${ }^{1}$, \\ Konstantinos Farsalinos ${ }^{1}$ (D) Panagiotis A. Eliopoulos ${ }^{2}(\mathbb{D})$ and Konstantinos Poulas ${ }^{1, *(1)}$ \\ 1 Department of Pharmacy, University of Patras, 26504 Patras, Greece; nikolaoskalyvas3@gmail.com (N.K.); \\ ntoukasaris7@gmail.com (A.N.); lagoipp@gmail.com (I.L.); kfarsalinos@gmail.com (K.F.) \\ 2 Department of Agrotechnology, University of Thessaly, Gaiopolis, 41500 Larissa, Greece; eliopoulos@uth.gr \\ * Correspondence: mantzoukas@upatras.gr (S.M.); kpoulas@upatras.gr (K.P.)
}

Received: 1 July 2020; Accepted: 11 September 2020; Published: 13 September 2020

\begin{abstract}
The present study investigates the interaction between cannabidiol (CBD) oil and three biopesticides: Azatin and two baculovirus formulations (Madex and Helicovex), both separately and in combination, in order to investigate their interaction against adults of four major coleopteran stored-product pests: Sitophilus zeamais (Coleoptera: Curculionidae), Rhyzopertha dominica (Coleoptera: Bostrichidae), Prostephanus truncatus (Coleoptera: Bostrichidae) and Trogoderma granarium (Coleoptera: Dermestidae). CBD, which has been understudied for its insecticidal properties, was applied at three different doses (500, 1500 and 3000 ppm). The biopesticides were administered at $1500 \mathrm{ppm}$. Interactions in the combined treatments were mathematically estimated as not synergistic and mostly competitive except for the combined treatments of CBD (1500 and $3000 \mathrm{ppm}$ ) with Azatin (1500 ppm) which were marked by an additive interaction. In its individual application, CBD oil generated the highest insect mortality while its effect was clearly dose-dependent. The findings reveal a promising effect of CBD oil against these coleopterans which had not been previously tested together.
\end{abstract}

Keywords: CBD oil; biopesticides; stored pests; insecticidal; Azatin; madex; helicovex

\section{Introduction}

Insect pests cause severe damage to plant life and crops with an estimation of approximately $30 \%$ of crop yields lost due to pathogens [1], rendering their control a crucial issue for farmers worldwide. Although chemical pesticides remain the most effective method for the management of serious storage pests, their action has proven to be hazardous for the user and the environment [2,3]. Moreover, their extensive and often unnecessary application has increased resistance to chemical pesticides in target species [4]. Appreciation for the health and environmental risks involved in the use of chemical pesticides has propelled a shift towards the search for biopesticides as well as botanicals with insecticidal and repellent effects. Botanical insecticides present an array of advantages including low mammalian toxicity, environmental safety, efficacy as well as a reduced likelihood to cause resistance in arthropods [5-7], and as such, they have legitimately attracted a high degree of interest for their pest control potential, including in the management of stored-product pests [8].

The use of entomopathogenic microorganisms is central in biological pest control [9-14]. Entomopathogenic viruses are among the microorganisms whose value has been acknowledged in biological pest control as they exhibit good insecticidal potential, high selectivity, making them safe for nontarget arthropods, and compatible with other pest control methods [15]. Cydia pomonella 
Granulovirus (CpGV) and Helicoverpa armigera Nucleopolyhedrovirus (HearNPV) are two baculoviruses with proven efficacy against Lepidopteran pests. Research has shown that HearNPV can be utilized as a control agent for pests which attack chickpea and sunflower crops [16,17]. Likewise, many plants can be used as biopesticides, as many plant species are famous for their insecticidal properties [18-21]. A widely used plant species is Azadirachta indica A. Juss (Sapindales: Meliaceae). Numerous studies highlight the unique properties of its extracted oil and its effectiveness as an insecticide [22-24]. Research has also been conducted over recent years on Cannabis sativa L. (Rosids: Cannabaceae) and, especially, one major cannabinoid that is present in the plant which is cannabidiol (CBD). Cannabis-based insecticides and repellents are widely available $[5,25]$; however, the mode of action of CBD as an insecticide or repellent is still under investigation.

Two or more biopesticides are often combined to create an enhanced product with a better efficiency or duration [26-28]. However, they may not coexist harmoniously in a mixture and their action is not always additive. Their interaction can be synergistic or antagonistic [29-35], depending on the concentration, species, mode of action, target organism, temperature conditions, and relative humidity (r.h) conditions of each biopesticide [33-47]. The combined use of two or more biopesticides in one mixture may result in less (or more) environmental harm or less (or more) harm to nontarget organisms [48-50]. Biopesticides based on plant essential oils and microorganisms are considered relatively safe and their efficacy has been demonstrated against different insect species.

The aim of the present study was to investigate the insecticidal effect of CBD oil in combination with three biopesticides: CBD + Madex (Cydia Pomonella Granulovirus (CpGV)), CBD + Azatin (Azadirachtin), and CBD + Helicovex (Helicoverpa Armigera Nucleopoyhedrovirus (HearNPV)). The above combinations were tested against adults of Sitophilus zeamais (Coleoptera: Curculionidae), Rhyzopertha dominica (Coleoptera: Bostrichidae), Prostephanus truncatus (Coleoptera: Bostrichidae), and Trogoderma granarium (Coleoptera: Dermestidae). This study is a follow up on previous similar studies investigating the synergism between biopesticides, and biopesticides and botanicals to enhance toxicity against stored-product pests [26-28]. CBD oil and these three biopesticides have never been investigated for their combined action against the four coleopteran pests. This is the first time CBD oil has been tested against the specific coleopteran pests.

\section{Materials and Methods}

\subsection{Insect Rearing}

Four major stored-product beetle species were selected: the maize weevil S. zeamais, the lesser grain borer $R$. dominica, the larger grain borer P. truncatus and the khapra beetle, T. granarium. These species cover a wide spectrum of globally distributed stored-product pests and cause serious losses and degradations to a wide range of commodities. All species were reared in a growth chamber (PHC Europe/Sanyo/Panasonic Biomedical MLR-352-PE) in controlled environmental conditions, at $27.5^{\circ} \mathrm{C}$ and $75 \%$ relative humidity (r.h.) in the Laboratory of Molecular Biology and Immunology, Department of Pharmacy, University of Patras. We used mixed sex adults, $<2$ weeks old. The bioassays were carried out on wheat (var. Mexa). This grain was adjusted at $12 \%$ moisture content (m.c.), via storage in ambient conditions, for $28 \mathrm{~d}$. Whole grain kernels were used because these species are known to infest sound grains.

\subsection{Bioassay}

Individual lots of $500 \mathrm{~g}$ of wheat were placed in $0.45 \mathrm{~L}$ cylindrical glass jars, in the laminar flow cabinet (Equip Vertical Air Laminar Flow Cabinet Clean Bench. Mechanical Application LTD. Athens. Greece). The CBD solution (3\% oil produced by Enecta Athens, Greece) was diluted with methanol $(\mathrm{MeOH})$ and was applied on the commodities at three concentrations (500, 1500 and $3000 \mathrm{ppm}$ ). The same process was followed for the biopesticides whereby Madex (Cydia pomonella Granulovirus (CpGV)) (Hellafarm, Athens, Greece), Azatin (Azadirachtin A 2.6 EC) (K\&NE Earth 
Matters, Thessaloniki, Greece), and Helicovex (Helicoverpa armigera Nucleopoyhedrovirus (HearNPV)) (Hellafarm, Athens, Greece) were tested at $1500 \mathrm{ppm}$. Solutions were sprayed on the products using the Potter spray tower (Burkard Manufacturing Co. Ltd., Rickmansworth, Hertfordshire, U.K.) at $1 \mathrm{kgf} \mathrm{cm}^{-2}$. The entire process was repeated twenty times, whereby new groups of insects were prepared each time. After the solutions had been sprayed on the product, the wheat lots were placed back into the jars and were shaken manually for $30 \mathrm{~s}$ to achieve an equal distribution of the treatment. The product was then air dried for 30 min especially for the CBD oil concentrations because methanol evaporates within a short time. Separate lots of wheat were sprayed with distilled water and $\mathrm{MeOH}$, to serve as controls. From each jar, ten $20 \mathrm{~g}$ samples were taken and placed in cylindrical plastic vials $(3 \mathrm{~cm}$ in diameter, $8 \mathrm{~cm}$ in height). The vials had a plastic lid with a hole in the center covered with fine mesh, while the internal "neck" of the vials was covered with Fluon (Northern Products, Woonsocket, RI USA), to prevent adult insects from escaping. Twenty individuals were placed within each vial (separate vials for each species) and the vials were placed in plastic boxes with saturated solutions of sodium chloride to maintain $75 \%$ r.h. Two hundred coleopteran adults were used for each concentration ( 20 adults per replication) and the experiment was replicated ten times. All boxes were then placed in incubators set at $27.5^{\circ} \mathrm{C}$ and $75 \%$ r.h. Adults were observed daily, and mortality was recorded at 7, 14, 21 and 28 days after treatment.

To study the combined effect of the treatments, adult insects were treated with a combination of each biopesticide with a CBD oil suspension, resulting in nine different combinations (Azatin $1500 \mathrm{ppm}$ + CBD 500 ppm, Azatin 1500 ppm + CBD 1500 ppm, Azatin 1500 ppm + CBD 3000 ppm; Madex 1500 ppm + CBD 500 ppm, Madex 1500 ppm + CBD 1500 ppm, Madex 1500 ppm + CBD 3000 ppm; Helicovex 1500 ppm + CBD 500 ppm, Helicovex 1500 ppm + CBD 1500 ppm, Helicovex 1500 ppm + CBD $3000 \mathrm{ppm}$ ). The product was initially sprayed with $2 \mathrm{~mL}$ of the CBD oil and, after $2 \mathrm{~s}$, with $2 \mathrm{~mL}$ of the biopesticide concentration. Twenty individuals were placed within each vial (separate vials for each species), and the vials were placed in plastic boxes with saturated solutions of sodium chloride to maintain $75 \%$ r.h. Replications and spray procedures for the combined treatments were carried out as in the individual treatments. Adults were observed daily, and mortality was recorded at 7, 14, 21 and 28 days after treatment.

\subsection{Mathematical Estimation}

The interaction between the pathogens was estimated using the formula of Robertson and Preisler [51]:

$$
P_{\mathrm{E}}=P_{0}+\left(1-P_{0}\right) \times\left(P_{1}\right)+\left(1-P_{0}\right) \times\left(1-P_{1}\right) \times\left(P_{2}\right)
$$

where $P_{\mathrm{E}}$ is the expected mortality induced by the combination of the two pathogens; $P_{0}$ is the observed mortality of the control; $P_{1}$ is the observed mortality caused by the first pathogen (separate action); $P_{2}$ is the observed mortality caused by the second pathogen (separate action). The distribution was determined by the chi-square formula:

$$
\mathrm{x}^{2}=\left(L_{0}-L_{\mathrm{E}}\right)^{2} / L_{\mathrm{E}}+\left(D_{0}-D_{\mathrm{E}}\right)^{2} / D_{\mathrm{E}}
$$

where $L_{0}$ is the number of recorded live larvae of the control. $D_{0}$ is the number of recorded dead larvae of the control, $L_{\mathrm{E}}$ is the expected number of live larvae, and $D_{\mathrm{E}}$ is the expected number of dead larvae (estimated the same as $P_{\mathrm{E}}$, with Equation (1)). The formula was used to test the hypothesis independent-simultaneous relationship $\left(d_{\mathrm{f}}=1, p=0.05\right)$. If $\mathrm{x}^{2}<3.84$, the ratio is defined as additive, if $x^{2}>3.84$ and the observed mortality is higher than expected, the relationship is defined as synergistic. On the contrary, if $x^{2}>3.84$ and the observed mortality is less than expected, the relationship is defined as competitive. 


\subsection{Statistical Analysis}

All values were arcsine transformed prior to the analysis to reduce the effect of the variance. Data were analyzed by a two-way ANOVA using the general linear model of the SPSS (version 25) (IBM 2019, Armonk, NY, USA). In case of significant F values, means were compared using the Bonferroni test. The Kaplan-Meier method of the SPSS (ver. 25) was also selected to determine the mean survival time of S. zeamais, R. dominica, P. truncatus and T. granarium adults following the application of the treatments. The Cox regression of the SPSS (ver. 25), a common survival analysis regression method that describes the relation between the event incidence and a set of covariates, was selected to determine the hazard effect of the individual and combined treatments.

\section{Results}

Essential oil and biopesticides induced significantly different levels of mortality on adults of S. zeamais, $R$. dominica, P. truncatus and T. granarium. Significant differences were recorded between treatment $\left(\mathrm{F}=108.272, d_{\mathrm{f}}=6.139, p<0.001\right)$ and replication $\left(\mathrm{F}=10.909, d_{\mathrm{f}}=4.139, p<0.001\right)$ but not between the coleopteran species of the experiment $\left(\mathrm{F}=1.911, d_{\mathrm{f}}=3.139, p=0.132\right)$ as factors, in relation to the dependent variable of mortality at 28 days. The two-way factor model of coleopteran species $\times$ treatment $\left(\mathrm{F}=0.9722, d_{\mathrm{f}}=18.139, p=0.001\right)$ and treatment $\times$ replication $\left(\mathrm{F}=4.950, d_{\mathrm{f}}=24.139\right.$, $p=0.001$ ) showed a significant effect in terms of the mortality of coleopteran adults at 28 days. The mean beetle mortality caused by the separate action of CBD Oil, Azatin, Helicovex and Madex is presented in Table 1. More specifically, 28 days after the treatment with CBD oil, the mortality of $S$. zeamais adults increased from 38\% (500 ppm) to 70\% (3000 ppm), of R. dominica from 44\% (500 ppm) to $84 \%$ (3000 ppm), of P. truncatus from 48\% (500 ppm) to $80 \%$ (3000 ppm), and of T. granarium from $48 \%$ (500 ppm) to 62\% (3000 ppm) (Table 1). At 28 days, Azatin-induced mortality to Coleoptera was between 38\% (T. granarium) and 50\% (P. truncatus); Helicovex-induced mortality was between 16\% (S. zeamais) and 20\% (all others) (Table 1); Madex-induced mortality was between 18\% (S. zeamais) and $22 \%$ (R. dominica) (Table 1).

A total of nine combined treatments of CBD oil with the biopesticides were applied against S. zeamais. R. dominica. P. truncatus and T. granarium. Significant differences were recorded between treatment $\left(\mathrm{F}=57.641, d_{f}=8.179, p<0.001\right)$ and between the tested insects of the experiment $(\mathrm{F}=14.149$, $\left.d_{\mathrm{f}}=3.179, p<0.001\right)$ as factors, in relation to the dependent variable of mortality, at 28 days. The adult mortality of the four beetles varied significantly in terms of the combinations at two-way factor insect * treatment $\left(\mathrm{F}=2.138, d_{\mathrm{f}}=24.179, p=0.003\right)$ at 28 days. After 28 days, the mean mortality of the combined treatments of CBD Oil, Azatin, Helicovex and Madex was, for S. zeamais adults treated with CBD and Azatin between 36\% (CBD 500-Azatin 1500 ppm) and 84\% (CBD 3000-Azatin 1500 ppm), with CBD and Helicovex between 30\% (CBD 500-Helicovex 1500 ppm) and 62\% (CBD 3000-Helicovex 1500 ppm), and with CBD and Madex between 18\% (CBD 500-Madex 1500 ppm) and 40\% (CBD 3000-Madex $1500 \mathrm{ppm}$ ) (Table 2); for R. dominica adults treated with CBD and Azatin, the mean mortality was between 50\% (CBD 500-Azatin 1500 ppm) and 90\% (CBD 3000-Azatin 1500 ppm), with CBD and Helicovex between 40\% (CBD 500-Helicovex 1500 ppm) and 70\% (CBD 3000-Helicovex 1500 ppm), and with CBD and Madex between 32\% (CBD 500-Madex 1500 ppm) and 62\% (CBD 3000-Madex 1500 ppm) (Table 2); for P. truncatus adults treated with CBD and Azatin, the mean mortality was between 44\% (CBD 500-Azatin 1500 ppm) and 94\% (CBD 3000-Azatin 1500 ppm), with CBD and Helicovex between 38\% (CBD 500-Helicovex 1500 ppm) and 70\% (CBD 3000-Helicovex 1500 ppm), and with CBD and Madex between 26\% (CBD 500-Madex 1500 ppm) and 52\% (CBD 3000-Madex $1500 \mathrm{ppm}$ ) (Table 2); for T. granarium adults treated with CBD and Azatin, the mean mortality was between 50\% (CBD 500-Azatin 1500 ppm) and 62\% (CBD 3000-Azatin 1500 ppm), with CBD and Helicovex between 46\% (CBD 500-Helicovex 1500 ppm) and 68\% (CBD 3000-Helicovex 1500 ppm), and with CBD and Madex between 38\% (CBD 500-Madex 1500 ppm) and 54\% (CBD 3000-Madex 1500 ppm) (Table 2). 
Table 1. Mean (\%) mortality and median survival time (days) of adults of S. zeamais, R. dominica, P. truncatus and T. granarium treated separately with cannabidiol (CBD) oil, Azatin, Helicovex and Madex after 28 days. Mean \% mortality for different concentrations or biopesticide, within the same insect and pathogen, followed by the same small letter are not significantly different. Mean \% mortality for different insect, within the same concentrations or biopesticide, followed by the same capital letter are not significantly different (Bonferroni test, $\mathrm{a}=0.05$ ). Estimate Median survival time (days) of the same insect followed by the same small letter are not significantly different (Kaplan-Meier, $\mathrm{a}=0.05$ ). * Median Survival Time (S. zeamais: F: $11.000 ; d_{\mathrm{f}}: 6 ; p=0.225, R$ dominica: F: $1.565 ; d_{\mathrm{f}}: 6 ; p=0.975$, P. truncatus: F: $1.259 ; d_{\mathrm{f}}: 6 ; p=0.877$, T. granarium: F: $\left.1.240 ; d_{\mathrm{f}}: 6 ; p=0.893\right)(n=200)$.

\begin{tabular}{|c|c|c|c|c|c|c|c|c|c|c|}
\hline \multirow{3}{*}{ Insect } & \multicolumn{4}{|c|}{ Concentration (ppm) } & \multicolumn{2}{|c|}{ Mortality } & \multicolumn{4}{|c|}{ Median Survival Time (Days) * } \\
\hline & \multirow{2}{*}{$\begin{array}{c}\text { CBD } \\
\text { Oil }\end{array}$} & \multirow[b]{2}{*}{ Azatin } & \multirow[b]{2}{*}{ Helicovex } & \multirow[b]{2}{*}{ Madex } & \multirow[b]{2}{*}{$(\%)$} & \multirow[b]{2}{*}{ Sd } & \multirow[b]{2}{*}{ Estimate } & \multirow[b]{2}{*}{ Sd } & \multicolumn{2}{|c|}{ 95\% Confidence Interval } \\
\hline & & & & & & & & & $\begin{array}{l}\text { Lower } \\
\text { Bound }\end{array}$ & $\begin{array}{l}\text { Upper } \\
\text { Bound }\end{array}$ \\
\hline \multirow{7}{*}{ S. zeamais } & 0 & 0 & 0 & 0 & 6 & 2.00 & $27.160 \mathrm{a}$ & 0.510 & 26.160 & 28.160 \\
\hline & 500 & 0 & 0 & 0 & $38 \mathrm{bA}$ & 3.90 & $22.260 \mathrm{~b}$ & 1.251 & 19.809 & 24.711 \\
\hline & 1500 & 0 & 0 & 0 & $54 \mathrm{dA}$ & 1.40 & $21.140 \mathrm{~b}$ & 1.184 & 18.819 & 23.461 \\
\hline & 3000 & 0 & 0 & 0 & $70 \mathrm{eA}$ & 10.0 & $17.920 \mathrm{c}$ & 1.274 & 15.424 & 20.416 \\
\hline & 0 & 1500 & 0 & 0 & $46 \mathrm{cA}$ & 1.40 & $23.800 \mathrm{~b}$ & 0.950 & 21.939 & 25.661 \\
\hline & 0 & 0 & 1500 & 0 & $16 \mathrm{aA}$ & 3.90 & $26.180 \mathrm{a}$ & 0.787 & 24.638 & 27.722 \\
\hline & 0 & 0 & 0 & 1500 & $18 \mathrm{aA}$ & 3.40 & $26.180 a$ & 0.690 & 24.827 & 27.533 \\
\hline \multirow{7}{*}{ R. dominica } & 0 & 0 & 0 & 0 & 6 & 2.00 & $27.300 \mathrm{a}$ & 0.4 & 26.500 & 28.100 \\
\hline & 500 & 0 & 0 & 0 & $44 \mathrm{bA}$ & 5.20 & $21.280 \mathrm{~b}$ & 1.266 & 18.798 & 23.762 \\
\hline & 1500 & 0 & 0 & 0 & $60 \mathrm{cA}$ & 2.20 & $20.720 b$ & 1.155 & 18.455 & 22.985 \\
\hline & 3000 & 0 & 0 & 0 & $84 \mathrm{dA}$ & 5.50 & $15.380 \mathrm{c}$ & 1.228 & 13.272 & 18.088 \\
\hline & 0 & 1500 & 0 & 0 & $44 \mathrm{bA}$ & 1.40 & $23.240 \mathrm{~b}$ & 1.062 & 21.159 & 25.321 \\
\hline & 0 & 0 & 1500 & 0 & $20 \mathrm{aA}$ & 5.10 & $25.480 \mathrm{~b}$ & 0.904 & 23.708 & 27.252 \\
\hline & 0 & 0 & 0 & 1500 & $22 \mathrm{aA}$ & 4.50 & $25.620 \mathrm{~b}$ & 0.794 & 24.064 & 27.176 \\
\hline \multirow{7}{*}{ P. truncatus } & 0 & 0 & 0 & 0 & 6 & 3.00 & $27.020 \mathrm{a}$ & 0.560 & 25.922 & 28.118 \\
\hline & 500 & 0 & 0 & 0 & $48 \mathrm{bA}$ & 3.50 & $21.140 \mathrm{~b}$ & 1.183 & 18.821 & 23.459 \\
\hline & 1500 & 0 & 0 & 0 & $58 \mathrm{bA}$ & 4.80 & $20.720 b$ & 1.262 & 18.246 & 23.194 \\
\hline & 3000 & 0 & 0 & 0 & $80 \mathrm{cA}$ & 10.0 & $15.920 \mathrm{c}$ & 1.229 & 13.871 & 18.689 \\
\hline & 0 & 1500 & 0 & 0 & $50 \mathrm{bA}$ & 4.20 & $22.960 \mathrm{~b}$ & 1.093 & 20.817 & 25.103 \\
\hline & 0 & 0 & 1500 & 0 & $20 \mathrm{aA}$ & 7.10 & $25.200 \mathrm{a}$ & 0.979 & 23.281 & 27.119 \\
\hline & 0 & 0 & 0 & 1500 & $20 \mathrm{aA}$ & 7.10 & $26.180 \mathrm{a}$ & 0.722 & 24.766 & 27.594 \\
\hline \multirow{7}{*}{ T. granarium } & 0 & 0 & 0 & 0 & 8 & 2.00 & $27.020 \mathrm{a}$ & 0.626 & 25.792 & 28.248 \\
\hline & 500 & 0 & 0 & 0 & $44 \mathrm{aA}$ & 5.20 & $21.700 \mathrm{~b}$ & 1.253 & 19.244 & 24.156 \\
\hline & 1500 & 0 & 0 & 0 & $54 \mathrm{bA}$ & 2.20 & $20.700 \mathrm{~b}$ & 1.163 & 18.420 & 22.980 \\
\hline & 3000 & 0 & 0 & 0 & $62 \mathrm{cA}$ & 2.30 & $19.600 \mathrm{c}$ & 1.224 & 17.201 & 21.999 \\
\hline & 0 & 1500 & 0 & 0 & $38 \mathrm{aA}$ & 4.50 & $23.800 \mathrm{~b}$ & 0.953 & 21.933 & 25.667 \\
\hline & 0 & 0 & 1500 & 0 & $20 \mathrm{aA}$ & 7.10 & $25.620 \mathrm{a}$ & 0.876 & 23.903 & 27.337 \\
\hline & 0 & 0 & 0 & 1500 & $24 \mathrm{aA}$ & 5.50 & $26.740 \mathrm{a}$ & 0.537 & 25.687 & 27.793 \\
\hline
\end{tabular}

The results of the combined treatments showed different types of interaction between the biopesticides and CBD oil. In the cases of $S$. zeamais, the interaction between the treatments was competitive except for one (Table 3). For P. truncatus, the interaction between the treatments was competitive except two, and for $R$ dominica, the interaction between the treatments was competitive except three (Table 3). An additive interaction was recorded for the combined treatments of the CBD oil (1500 and 3000 ppm) with Azatin (1500 ppm) for all above beetles (Table 3). By contrast, Helicovex and CBD oil marked an additive interaction against $T$. granarium at all three doses, and against $R$. dominica at one dose (Table 3). No synergistic relationship was recorded in any of the combinations (Table 3).

The six combined treatments which were overall toxic to $S$. zeamais, $R$. dominica, P. truncatus and T. granarium adults had positive $\beta$-values (Table 4). The combined treatments which exhibited a dose-dependent action were CBD 3000-Madex 1500 ppm, CBD 1500-Helicovex 1500 ppm, CBD 3000-Helicovex 1500 ppm, CBD 500-Azatin 1500 ppm, CBD 1500-Azatin 1500 ppm, and CBD 3000-Azatin 1500 ppm. The overall Hazard Rate (Exp (B)) for all combined treatments was higher than the control. Finally, the above treatments were not statistically significant, except for CBD 3000-Helicovex 1500 ppm, CBD 1500-Azatin 1500 ppm and CBD 3000-Azatin 1500 ppm with $p$ values of $<0.001,<0.001$ and $<0.001$, respectively (Table 4). The individual treatments which also had an effect on the overall mortality of $S$. zeamais, $R$ dominica, $P$. truncatus and $T$. granarium adults had positive $\beta$-values and included all three CBD oil concentrations; they were also statistically significant from the 
remaining individual treatments. The overall hazard rate (Exp (B)) for the CBD oil concentrations was higher than the control.

Table 2. Mean\% mortality and median survival time (days) adults of S. zeamais, R. dominica, P. truncatus and T. granarium treated with CBD oil in combination with Azatin, Helicovex and Madex. Mean\% mortality for different concentrations or biopesticide, within the same insect and pathogen, followed by the same small letter are not significantly different; mean \% mortality for different insect, within the same concentrations or biopesticide, followed by the same capital letter are not significantly different (Bonferroni test, $\mathrm{a}=0.05$ ). Estimate median survival time (days) of the same insect followed by the same small letter are not significantly different (Kaplan-Meier, a $=0.05) .{ }^{*}$ Median survival time (S. zeamais: F: $0.207 ; d_{\mathrm{f}}: 8 ; p=0.936, R$ dominica: F: $0.565 ; d_{\mathrm{f}}: 8 ; p=0.975$, P. truncatus: F: $3.667 ; d_{\mathrm{f}}: 8 ; p=0.230$, T. granarium: $\left.\mathrm{F}: 0.125 ; d_{\mathrm{f}}: 8 ; p=0.975\right)$.

\begin{tabular}{|c|c|c|c|c|c|c|c|c|c|c|}
\hline \multirow{3}{*}{ Insect } & \multicolumn{4}{|c|}{ Combined Concentration (ppm) } & \multicolumn{2}{|c|}{ Mortality } & \multicolumn{4}{|c|}{ Median Survival Time (Days) * } \\
\hline & \multirow[b]{2}{*}{$\begin{array}{c}\text { CBD } \\
\text { Oil }\end{array}$} & \multirow[b]{2}{*}{ Azatin } & \multirow[b]{2}{*}{ Helicovex } & \multirow[b]{2}{*}{ Madex } & \multirow[b]{2}{*}{$(\%)$} & \multirow[b]{2}{*}{ Sd } & \multirow[b]{2}{*}{ Estimate } & \multirow[b]{2}{*}{ Sd } & \multicolumn{2}{|c|}{ 95\% Confidence Interval } \\
\hline & & & & & & & & & $\begin{array}{l}\text { Lower } \\
\text { Bound }\end{array}$ & $\begin{array}{l}\text { Upper } \\
\text { Bound }\end{array}$ \\
\hline \multirow{9}{*}{ S. zeamais } & 500 & 1500 & 0 & 0 & $36 \mathrm{aA}$ & 4.90 & $25.060 \mathrm{a}$ & 0.793 & 23.506 & 26.614 \\
\hline & 1500 & 1500 & 0 & 0 & $68 \mathrm{cA}$ & 1.98 & $20.440 \mathrm{~b}$ & 1.041 & 18.399 & 22.481 \\
\hline & 3000 & 1500 & 0 & 0 & $84 \mathrm{dA}$ & 3.48 & $15.400 \mathrm{c}$ & 1.235 & 12.979 & 17.821 \\
\hline & 500 & 0 & 1500 & 0 & $30 \mathrm{aA}$ & 5.00 & $24.360 \mathrm{a}$ & 1.009 & 22.382 & 26.338 \\
\hline & 1500 & 0 & 1500 & 0 & $46 \mathrm{bA}$ & 5.48 & $23.080 \mathrm{a}$ & 0.953 & 21.212 & 24.948 \\
\hline & 3000 & 0 & 1500 & 0 & $62 \mathrm{cA}$ & 1.37 & $21.000 \mathrm{~b}$ & 1.156 & 18.734 & 23.266 \\
\hline & 500 & 0 & 0 & 1500 & $18 \mathrm{aA}$ & 4.37 & $25.900 \mathrm{a}$ & 0.727 & 24.475 & 27.325 \\
\hline & 1500 & 0 & 0 & 1500 & $22 \mathrm{aA}$ & 3.04 & $25.060 \mathrm{a}$ & 0.954 & 23.191 & 26.929 \\
\hline & 3000 & 0 & 0 & 1500 & $40 \mathrm{aA}$ & 1.07 & $22.960 \mathrm{~b}$ & 1.095 & 20.814 & 25.106 \\
\hline \multirow{9}{*}{$R$ dominica } & 500 & 1500 & 0 & 0 & $50 \mathrm{bA}$ & 10.0 & $22.680 \mathrm{a}$ & 1.020 & 20.681 & 24.679 \\
\hline & 1500 & 1500 & 0 & 0 & $80 \mathrm{dA}$ & 5.80 & $18.060 \mathrm{a}$ & 1.081 & 15.941 & 20.179 \\
\hline & 3000 & 1500 & 0 & 0 & $90 \mathrm{eB}$ & 1.07 & $14.140 \mathrm{~b}$ & 1.176 & 11.835 & 16.445 \\
\hline & 500 & 0 & 1500 & 0 & $40 \mathrm{bA}$ & 5.80 & $23.380 \mathrm{a}$ & 1.087 & 21.250 & 25.510 \\
\hline & 1500 & 0 & 1500 & 0 & $60 \mathrm{cA}$ & 2.25 & $22.260 \mathrm{a}$ & 1.079 & 20.145 & 24.375 \\
\hline & 3000 & 0 & 1500 & 0 & $70 \mathrm{cA}$ & 1.07 & $19.880 \mathrm{a}$ & 1.195 & 17.539 & 22.221 \\
\hline & 500 & 0 & 0 & 1500 & $32 \mathrm{aB}$ & 4.40 & $23.520 a$ & 1.072 & 21.419 & 25.621 \\
\hline & 1500 & 0 & 0 & 1500 & $42 \mathrm{bB}$ & 2.30 & $22.820 \mathrm{a}$ & 1.089 & 20.686 & 24.954 \\
\hline & 3000 & 0 & 0 & 1500 & $60 \mathrm{cB}$ & 4.14 & $21.000 \mathrm{a}$ & 1.174 & 18.699 & 23.301 \\
\hline \multirow{9}{*}{ P. truncatus } & 500 & 1500 & 0 & 0 & $44 \mathrm{dA}$ & 2.37 & $23.520 \mathrm{a}$ & 1.008 & 21.544 & 25.496 \\
\hline & 1500 & 1500 & 0 & 0 & $74 \mathrm{fA}$ & 5.17 & $19.320 \mathrm{a}$ & 1.072 & 17.218 & 21.422 \\
\hline & 3000 & 1500 & 0 & 0 & $94 \mathrm{gB}$ & 5.40 & $13.300 \mathrm{~b}$ & 1.119 & 11.107 & 15.493 \\
\hline & 500 & 0 & 1500 & 0 & $38 \mathrm{cA}$ & 2.37 & $23.520 \mathrm{a}$ & 1.072 & 21.419 & 25.621 \\
\hline & 1500 & 0 & 1500 & 0 & $54 \mathrm{eB}$ & 1.40 & $22.960 \mathrm{a}$ & 1.049 & 20.904 & 25.016 \\
\hline & 3000 & 0 & 1500 & 0 & $70 \mathrm{fA}$ & 3.07 & $26.765 a$ & 1.198 & 24.416 & 29.114 \\
\hline & 500 & 0 & 0 & 1500 & $26 a B$ & 1.40 & $24.220 \mathrm{a}$ & 1.055 & 22.152 & 26.288 \\
\hline & 1500 & 0 & 0 & 1500 & $30 \mathrm{bC}$ & 2.25 & $24.220 \mathrm{a}$ & 1.029 & 22.203 & 26.237 \\
\hline & 3000 & 0 & 0 & 1500 & $52 \mathrm{eC}$ & 3.04 & $21.000 \mathrm{a}$ & 1.177 & 18.692 & 23.308 \\
\hline \multirow{9}{*}{ T. granarium } & 500 & 1500 & 0 & 0 & $50 \mathrm{bA}$ & 1.07 & $22.680 \mathrm{a}$ & 1.061 & 20.601 & 24.759 \\
\hline & 1500 & 1500 & 0 & 0 & $56 \mathrm{bB}$ & 2.94 & $21.420 \mathrm{a}$ & 1.183 & 19.102 & 23.738 \\
\hline & 3000 & 1500 & 0 & 0 & $62 c C$ & 2.37 & $20.580 a$ & 1.213 & 18.203 & 22.957 \\
\hline & 500 & 0 & 1500 & 0 & $46 \mathrm{aA}$ & 3.48 & $22.820 \mathrm{a}$ & 1.105 & 20.654 & 24.986 \\
\hline & 1500 & 0 & 1500 & 0 & $54 \mathrm{bA}$ & 3.40 & $22.260 \mathrm{a}$ & 1.136 & 20.034 & 24.486 \\
\hline & 3000 & 0 & 1500 & 0 & $68 \mathrm{cA}$ & 2.37 & $20.440 \mathrm{a}$ & 1.220 & 18.049 & 22.831 \\
\hline & 500 & 0 & 0 & 1500 & $38 \mathrm{aB}$ & 5.95 & $23.660 \mathrm{a}$ & 1.074 & 21.554 & 25.766 \\
\hline & 1500 & 0 & 0 & 1500 & $48 \mathrm{aD}$ & 3.37 & $23.52 \mathrm{a} 0$ & 1.006 & 21.548 & 25.492 \\
\hline & 3000 & 0 & 0 & 1500 & $54 \mathrm{bC}$ & 3.48 & $22.680 \mathrm{a}$ & 1.059 & 20.604 & 24.756 \\
\hline
\end{tabular}


Table 3. Observed and expected mortality of S. zeamais, R. dominica, P. truncatus and T. granarium adults at the end of the experiment (28 days) treated with CBD oil in combinations with Azatin, Helicovex and Madex, plus their interaction (A = Additive. $C=$ Competitive. $S=$ Synergistic) $(n=200)$. ${ }^{*}$ Expected mortality is calculated according to Robertson and Preisler [51].

\begin{tabular}{|c|c|c|c|c|c|c|c|}
\hline \multicolumn{4}{|c|}{ Combined Concentration (ppm) } & \multicolumn{2}{|c|}{ Mortality (\%) } & \multirow[t]{2}{*}{$\begin{array}{c}\chi^{2} \\
\left(1 d_{\mathrm{f}} ; p=0.05\right)\end{array}$} & \multirow[t]{2}{*}{ Interaction } \\
\hline $\begin{array}{c}\text { CBD } \\
\text { Oil }\end{array}$ & Azatin & Helicovex & Madex & Observed & Expected * & & \\
\hline \multicolumn{8}{|c|}{ S. zeamais } \\
\hline 500 & 1500 & 0 & 0 & 36 & 68 & 24.53 & C \\
\hline 1500 & 1500 & 0 & 0 & 68 & 76 & 14.33 & $\mathrm{C}$ \\
\hline 3000 & 1500 & 0 & 0 & 84 & 84 & 0.023 & A \\
\hline 500 & 0 & 1500 & 0 & 30 & 51 & 8.864 & $\mathrm{C}$ \\
\hline 1500 & 0 & 1500 & 0 & 46 & 64 & 7.512 & $\mathrm{C}$ \\
\hline 3000 & 0 & 1500 & 0 & 62 & 76 & 6.224 & $\mathrm{C}$ \\
\hline 500 & 0 & 0 & 1500 & 18 & 52 & 23.452 & $\mathrm{C}$ \\
\hline 1500 & 0 & 0 & 1500 & 22 & 64 & 39.543 & $\mathrm{C}$ \\
\hline 3000 & 0 & 0 & 1500 & 40 & 76 & 38.247 & C \\
\hline \multicolumn{8}{|c|}{$R$ dominica } \\
\hline 500 & 1500 & 0 & 0 & 50 & 70 & 10.129 & C \\
\hline 1500 & 1500 & 0 & 0 & 80 & 78 & 0.033 & $\mathrm{~A}$ \\
\hline 3000 & 1500 & 0 & 0 & 90 & 91 & 0.161 & $\mathrm{~A}$ \\
\hline 500 & 0 & 1500 & 0 & 40 & 57 & 6.562 & $\mathrm{C}$ \\
\hline 1500 & 0 & 1500 & 0 & 60 & 69 & 2.339 & A \\
\hline 3000 & 0 & 1500 & 0 & 70 & 87 & 15.251 & $\mathrm{C}$ \\
\hline 500 & 0 & 0 & 1500 & 32 & 58 & 14.995 & $\mathrm{C}$ \\
\hline 1500 & 0 & 0 & 1500 & 42 & 70 & 19.831 & $\mathrm{C}$ \\
\hline 3000 & 0 & 0 & 1500 & 60 & 88 & 38.586 & $\mathrm{C}$ \\
\hline \multicolumn{8}{|c|}{ P. truncatus } \\
\hline 500 & 1500 & 0 & 0 & 44 & 75 & 26.968 & C \\
\hline 1500 & 1500 & 0 & 0 & 74 & 80 & 1.236 & $\mathrm{~A}$ \\
\hline 3000 & 1500 & 0 & 0 & 94 & 90 & 0.678 & $\mathrm{~A}$ \\
\hline 500 & 0 & 1500 & 0 & 38 & 60 & 11.007 & $\mathrm{C}$ \\
\hline 1500 & 0 & 1500 & 0 & 54 & 68 & 4.808 & $\mathrm{C}$ \\
\hline 3000 & 0 & 1500 & 0 & 70 & 84 & 8.757 & $\mathrm{C}$ \\
\hline 500 & 0 & 0 & 1500 & 26 & 60 & 25.568 & $\mathrm{C}$ \\
\hline 1500 & 0 & 0 & 1500 & 30 & 68 & 34.148 & $\mathrm{C}$ \\
\hline 3000 & 0 & 0 & 1500 & 52 & 85 & 42.509 & $\mathrm{C}$ \\
\hline \multicolumn{8}{|c|}{ T. granarium } \\
\hline 500 & 1500 & 0 & 0 & 50 & 68 & 7.499 & C \\
\hline 1500 & 1500 & 0 & 0 & 56 & 73 & 8.150 & $\mathrm{C}$ \\
\hline 3000 & 1500 & 0 & 0 & 62 & 78 & 3.139 & A \\
\hline 500 & 0 & 1500 & 0 & 46 & 58 & 3.372 & A \\
\hline 1500 & 0 & 1500 & 0 & 54 & 66 & 3.292 & $\mathrm{~A}$ \\
\hline 3000 & 0 & 1500 & 0 & 68 & 72 & 0.403 & A \\
\hline 500 & 0 & 0 & 1500 & 38 & 60 & 10.952 & $\mathrm{C}$ \\
\hline 1500 & 0 & 0 & 1500 & 48 & 67 & 9.017 & $\mathrm{C}$ \\
\hline 3000 & 0 & 0 & 1500 & 54 & 73 & 9.675 & $\mathrm{C}$ \\
\hline
\end{tabular}


Table 4. Toxicity variables in the equation from Cox regression for the treatments against S. zeamais, R. dominica, P. truncatus and T. granarium adults at the end of Table. 28 days. ${ }^{\dagger} \mathrm{B}$ : B values are associated with increased hazard and decreased survival time; as the predictor increases, the hazard of the event increases and the predicted survival duration decreases. Negative coefficients indicate decreased hazard and increased survival times. ${ }^{+\dagger} \operatorname{Exp}(\mathrm{B})$ : the ratio of hazard rates.

\begin{tabular}{|c|c|c|c|c|c|c|}
\hline \multicolumn{7}{|c|}{ Variables in the Equation } \\
\hline \multirow{2}{*}{$\begin{array}{l}\text { Combined Concentration } \\
\text { (ppm) }\end{array}$} & \multirow{2}{*}{$\mathbf{B}^{+}$} & \multirow{2}{*}{ Sd } & \multirow{2}{*}{ Sig. } & \multirow{2}{*}{$\operatorname{Exp}(B)^{\dagger+}$} & \multicolumn{2}{|c|}{$95.0 \%$ CI for $\operatorname{Exp}(\mathrm{B})$} \\
\hline & & & & & Lower & Upper \\
\hline CBD 500-Madex 1500 & -0.354 & 0.167 & 0.034 & 0.702 & 0.506 & 0.973 \\
\hline CBD 1500-Madex 1500 & -0.217 & 0.160 & 0.176 & 0.805 & 0.588 & 1.102 \\
\hline CBD 3000-Madex 1500 & 0.254 & 0.146 & 0.082 & 1.289 & 0.968 & 1.716 \\
\hline CBD 500-Helicovex 1500 & -0.113 & 0.157 & 0.471 & 0.893 & 0.657 & 1.215 \\
\hline CBD 1500-Helicovex 1500 & 0.235 & 0.145 & 0.106 & 1.265 & 0.951 & 1.681 \\
\hline CBD 3000-Helicovex 1500 & 0.593 & 0.138 & 0.000 & 1.810 & 1.381 & 2.373 \\
\hline CBD 500-Azatin 1500 & 0.055 & 0.150 & 0.713 & 1.057 & 0.787 & 1.419 \\
\hline CBD 1500-Azatin 1500 & 0.670 & 0.137 & 0.000 & 1.955 & 1.495 & 2.556 \\
\hline CBD 3000-Azatin 1500 & 1.088 & 0.133 & 0.000 & 2.969 & 2.288 & 3.853 \\
\hline \multicolumn{7}{|l|}{$\begin{array}{l}\text { Separately Concentration } \\
(\text { (ppm) }\end{array}$} \\
\hline CBD 500 & 0.082 & 0.153 & 0.007 & 1.085 & 0.804 & 1.465 \\
\hline CBD 1500 & 0.396 & 0.143 & 0.000 & 1.485 & 1.123 & 1.964 \\
\hline CBD 3000 & 0.858 & 0.136 & 0.000 & 2.359 & 1.807 & 3.080 \\
\hline Control & -1.964 & 0.288 & 0.830 & 0.140 & 0.080 & 0.247 \\
\hline Helicovex 1500 & -0.906 & 0.195 & 0.410 & 0.404 & 0.276 & 0.592 \\
\hline Madex 1500 & -0.928 & 0.215 & 0.592 & 0.396 & 0.259 & 0.603 \\
\hline Azatin 1500 & -0.879 & 0.191 & 0.315 & 0.415 & 0.285 & 0.604 \\
\hline
\end{tabular}

\section{Discussion}

The present study represents an investigation of the individual and combined insecticidal effect of CBD oil and three biopesticides, Azatin, Madex and Helicovex. In our study, the combined treatments of $\mathrm{CBD}$ with the biopesticides produced varying results. Our hypothesis was that the interaction between treatments would be more additive than synergistic. Insects would thus die from a reinforced action. Our results support this hypothesis in the case of CBD with Azatin for all tested insects, as well as in the case of two insects with CBD and Helicovex. Overall, no combination exhibited synergism.

On its own, CBD oil yielded the highest mortality rates at 28 days. Its action was dose-dependent which is generally the case with essential oils, whose effects include metabolic and physiological disruptions, neurotoxic effects, and histological changes [29]. Benelli et al. [5] report that the essential oil from the inflorescence of industrial hemp was highly toxic to aphids and flies, although results ranged from moderate to scarcely toxicity larvae and adults. C. sativa $\mathrm{L}$ and CBD-related studies on their insecticidal properties are scarce. In most studies, C. sativa was tested as a companion plant, harvested plant material, in aqueous and solvent extracts, and as essential oil [25]. There is bound to be variation in the findings concerning the insecticidal effects of the plant. In their review, McPartland and Sheikh [25] concluded that of all bioassays, the essential oil produced the best results in the treated insects which were mainly thin-cuticle small arthropods.

Azadin (1500 ppm) generated moderate to low mortality reaching a peak at 50\% (P. truncatus) [30]. Similarly, albeit as a crude powder, A. indica was tested against $P$. truncatus and was found to be weakly toxic to the insect, causing a mere mortality of $40 \%$ at 28 days [30]. In a similar vein, azadirachtin-enriched neem kernel extracts on wheat consumed by $R$. dominica did not have any effect on parent mortality although they inhibited $F_{1}$ progeny by $98 \%$ [47]. Tofel et al. [48] found Azadirachtin seed oil to be lethal to Callosobruschus maculatus Fabricius (Coleoptera: Chrysomelidae) and S. zeamais adults. Variation in the effect of azadirachtin and neem extracts may be the product of 
differences in the derivatives and formulations used as well as of a complex of several other factors such as grain type, target species, dosage, temperature, humidity and others [49]. In the present study, moderate to low mortality could be interpreted as an indirect event resulting from the disruption in the feeding of the insect, drawing on Xie et al. [51] in their interpretation of the killing of Sitophilus oryzae Linnaeus (Coleoptera: Curculionidae), Tribolium castaneum Herbst (Coleoptera: Tenebrionidae) and Cryptolestes ferrugineus Ganglbauer (Coleoptera: Laemophloeidae).

Finally, Madex and Helicovex were ineffective as mortality rates did not exceed $22 \%$ at 28 days. The low mortality can be accounted for by the age of the insects, as viruses are mostly pathogenic to larvae whereby molting and pupation is blocked by the infection [52], and by the different insect order as the two baculoviruses are mostly effective against Lepidopterans. Moreover, they were slow in their action, which is a known disadvantage of viruses in general [53].

Examining how pesticides interact with each other advances research on to the creation of more effective, cost efficient and lasting pesticidal products. Interaction is determined by the interplay of many factors such as the compound itself, dose, insect species, and others. One or all individual components of a combination may enhance (synergism) or suppress (antagonism/competition) each other's toxicity, or simply remain within the additive framework [54-57]. The observed additive effect with high toxicity values ( $\mathrm{B}$ and $\operatorname{Exp}(\mathrm{B})$ ) is the result of the Azadirachtin making insects more sensitive to $\mathrm{CBD}$, thus adding to the result.

It is also worth pointing out that $T$. granarium (all concentrations) and $R$. dominica (CBD 1500-Helicovex 1500 ppm) were the only beetles in which CBD and the Helicovex did not compete, whereas CBD had a negative interaction with Madex or Helicovex in the case of the other two Coleoptera. Thus, an additive effect with very good toxicity values (B and Exp (B)) was shown when adults of T. granarium and R. dominica were treated with combined concentrations. The T. granarium and $R$. dominica cases are a finding worth exploring further.

On the contrary, the mathematical estimation revealed that most combinations were competitive. A competitive interaction refers to the negative relationship between pathogens. The nature of competition between tested treatments is not documented. In our case, the combined use of CBD with Madex exhibited negative B values but good toxicity values. The combined concentration of CBD with Madex did not produce increased hazard and decreased survival time in terms of the mortality of the beetles.

\section{Conclusions}

Although acute toxicity of plant-derived substances is lower than that of their chemical counterparts, they have attracted a high degree of interest for their pest control potential, including for the management of stored-product pests. As research in biological control is gaining considerable momentum, and as the list of entomopathogenic properties of plants and biopesticides is continually enriched, our findings highlight the potential of CBD against major stored-product pests and add to the limited literature concerning this essential oil.

Author Contributions: Conceptualization, S.M. and K.P.; methodology, S.M.; software, S.M.; validation, S.M., P.A.E., I.L., and K.P.; formal analysis, S.M., I.L., and A.N.; investigation, S.M., N.K., I.L., and A.N.; resources, K.P.; data curation, S.M.; writing-original draft preparation, S.M., A.N., K.F., P.A.E., and K.P.; writing一review and editing, S.M., P.A.E. and K.P.; visualization, S.M.; supervision, K.P.; project administration, K.P.; funding acquisition, K.P. All authors have read and agreed to the published version of the manuscript.

Funding: We acknowledge support of this work by the project "Synthetic Biology: From omics technologies to genomic engineering (OMIC-ENGINE)" (MIS 5002636), which is implemented under the Action "Reinforcement of the Research and Innovation Infrastructure", funded by the Operational Programme "Competitiveness, Entrepreneurship and Innovation" (NSRF 2014-2020) and co-financed by Greece and the European Union (European Regional Development Fund).

Acknowledgments: We would like to thank Ioanna Zampara in interpreting and translating, for her help with the language. Authors are also grateful to the National Research Infrastructure Program of the University of Patras "OMIC-ENGINE-Synthetic Biology: from omics technologies to genomic engineering" for financial support of the personnel. 
Conflicts of Interest: The authors declare no conflict of interest.

\section{References}

1. Becker-Ritt, A.B.; Carlini, C.R. Fungitoxic and insecticidal plant polypeptides. Biopolymers 2012, 98, 367-384. [CrossRef] [PubMed]

2. Diaz, J.H. Chemical and plant-based insect repellents: Efficacy, safety, and toxicity. Wilderness Environ. Med. 2016, 27, 153-163. [CrossRef] [PubMed]

3. Jiang, W.; Hernandez, B.; Richmond, D.; Yanga, N. Harvesters in strawberry fields: A literature review of pesticide exposure, an observation of their work activities, and a model for exposure prediction. J. Expo. Sci. Environ. Epidemiol. 2017, 27, 391-397. [CrossRef] [PubMed]

4. Collins, P.J.; Schlipalius, D.I. Insecticide resistance. In Recent Advances in Stored Product Protection; Athanassiou, C.G., Arthur, F.H., Eds.; Springer: Berlin/Heidelberg, Germany, 2018; pp. 169-182.

5. Benelli, G.; Pavela, R.; Petrelli, R.; Cappellaci, L.; Santini, G.; Fiorini, D.; Sut, S.; Dall'Acqua, S.; Canale, A.; Maggi, F. The essential oil from industrial hemp (Cannabis sativa L.) by-products as an effective tool for insect pest management in organic crops. Ind. Crop. Prod. 2018, 122, 308-315. [CrossRef]

6. Pavela, R.; Benelli, G. Essential oils as eco-friendly biopesticides? Challenges and consraints. Trends Plant Sci. 2016, 21, 1000-1007. [CrossRef] [PubMed]

7. Said, P.; Pashte, V. Botanicals: The protectants of stored grains pests. Biosci. Trends 2015, 8, 3750-3755.

8. Spochacz, M.; Chowański, S.; Walkowiak-Nowicka, K.; Szymczak, M.; Adamski, Z. Plant-derived substances used against beetles-pests of stored crops and food-and their mode of action: A review. Compr. Rev. Food Sci. Food Saf. 2018, 17, 1339-1366. [CrossRef]

9. Kumar, K.K.; Sridhar, J.; Murali-Baskaran, R.K.; Senthil-Nathan, S.; Kaushal, P.; Dara, S.K.; Arthurs, S. Microbial biopesticides for insect pest management in India: Current status and future prospects. J. Invertebr. Pathol. 2019, 165, 74-81. [CrossRef]

10. Hatting, J.L.; Moore, S.D.; Malan, A.P. Microbial control of phytophagous invertebrate pests in South Africa: Current status and future prospects. J. Invertebr. Pathol. 2019, 165, 54-66. [CrossRef]

11. Lovett, B.; St. Leger, R.J. Genetically engineering better fungal biopesticides. Pest Manag. Sci. 2018, 74, 781-789. [CrossRef]

12. Beck, J.J.; Vannette, R.L. Harnessing insect-microbe chemical communications to control insect pests of agricultural systems. J. Agric. Food Chem. 2017, 65, 23-28. [CrossRef] [PubMed]

13. Carpio, C.; Dangles, O.; Dupas, S.; Léry, X.; López-Ferber, M.; Orbe, K.; Páez, D.; Rebaudo, F.; Santillán, A.; Yangari, B.; et al. Development of a viral biopesticide for the control of the Guatemala potato tuber moth Tecia solanivora. J. Invertebr. Pathol. 2013, 112, 184-191. [CrossRef] [PubMed]

14. Arthurs, S.; Dara, S.K. Microbial biopesticides for invertebrate pests and their markets in the United States. J. Invertebr. Pathol. 2019, 165, 13-21. [CrossRef] [PubMed]

15. Raymond, B.; Sayyed, A.H.; Wright, D.J. The compatibility of a nucleopolyhedrosis virus control with resistance management for Bacillus thuringiensis: Co-infection and cross-resistance studies with the diamondback moth, Plutella xylostella. J. Invertebr. Pathol. 2006, 93, 114-120. [CrossRef] [PubMed]

16. Jaques, R.P.; Hardman, J.M.; Laing, J.E.; Smith, R.E.; Bent, E. Orchard trials in Canada on control of Cydia Pomonella (Lep: Tortricidae) by granulosis Virus. Entomophaga 1994, 39, 281-292. [CrossRef]

17. Sun, X. History and current status of development and use of viral insecticides in China. Viruses 2015, 7, 306-319. [CrossRef]

18. Rajashekar, Y.; Bakthavatsalam, N.; Shivanandappa, T. Botanicals as grain protectants. Psyche 2012, 2012, 646740. [CrossRef]

19. Omar, K.; Faraj, N.M.; Malik, S.A.A.; Al-Farhani, I.M. Effect of some medicinal plants extracts and cypermthrin against Khapra Beetle (Trogoderma granarium Everts). Emir. J. Food Agric. 2012, 24, 120-127.

20. Maia, M.F.; Moore, S.J. Plant-based insect repellents: A review of their efficacy, development, and testing. Malar. J. 2011, 10 (Suppl. 1), S11. [CrossRef]

21. Athanassiou, C.G.; Kavallieratos, N.G.; Evergetis, E.; Katsoula, A.-M.; Haroutounian, S.A. Insecticidal Efficacy of Silica Gel with Juniperus oxycedrus ssp. oxycedrus (Pinales: Cupressaceae) Essential Oil Against Sitophilus oryzae (Coleoptera: Curculionidae) and Tribolium confusum (Coleoptera: Tenebrionidae). J. Econ. Entomol. 2013, 106, 1902-1910. [CrossRef] 
22. Gupta, S.C.; Prasad, S.; Tyagi, A.K.; Kunnumakkara, A.B.; Aggarwal, B.B. Neem (Azadirachta indica): An indian traditional panacea with modern molecular basis. Phytomedicine 2017, 34, 14-20. [CrossRef] [PubMed]

23. Benelli, G.; Canale, A.; Toniolo, C.; Higuchi, A.; Murugan, K.; Pavela, R.; Nicoletti, M. Neem (Azadirachta indica): Towards the ideal insecticide? Nat. Prod. Res. 2017, 31, 369-386. [CrossRef] [PubMed]

24. Pascoli, M.; Jacques, M.T.; Agarrayua, D.A.; Avila, D.S.; Lima, R.; Fraceto, L.F. Neem oil based nanopesticide as an environmentally-friendly formulation for applications in sustainable agriculture: An ecotoxicological perspective. Sci. Total Environ. 2019, 677, 57-67. [CrossRef] [PubMed]

25. McPartland, J.M.; Sheikh, Z. A Review of Cannabis sativa-Based Insecticides, Miticides, and Repellents. J. Entomol. Zool. Stud. 2018, 6, 1288-1299. Available online: https://www.cabdirect.org (accessed on 6 July 2020).

26. Mascarin, G.M.; Delalibera, I. Insecticidal activity of the granulosis virus in combination with neem products and talc powder against the potato tuberworm Phthorimaea operculella (Zeller) (Lepidoptera: Gelechiidae). Neotrop. Entomol. 2012, 41, 223-231. [CrossRef]

27. Awan, D.A.; Ahmad, F.; Saleem, M.A.; Shakoori, A.R. Synergistic effect of piperonyl butoxide and emamectin benzoate on enzymatic activities in resistant populations of red flour beetle, Tribolium castaneum Herbst (Coleoptera: Tenebrionidae). Environ. Sci. Pollut. Res. 2019, 26, 14200-14213. [CrossRef]

28. Mwila, K.; Burton, M.H.; Van Dyk, J.S.; Pletschke, B.I. The effect of mixtures of organophosphate and carbamate pesticides on acetylcholinesterase and application of chemometrics to identify pesticides in mixtures. Environ. Monit. Assess. 2013, 185, 2315-2327. [CrossRef]

29. Campolo, O.; Giunti, G.; Russo, A.; Palmeri, V.; Zappalà, L. Essential oils in stored product insect pest control. J. Food Qual. 2018, 2018, 6906105. [CrossRef]

30. Chebet, F.; Deng, A.; Ogendo, J.O.; Kamau, A.; Bett, P. Bioactivity of selected plant powders against Prostephanus truncatus (Coleoptera: Bostrichidae) in stored maize grains. Plant Prot. Sci. 2013, 49, 34-43. [CrossRef]

31. Faraone, N.; Hillier, N.K.; Cutler, G.C. Plant essential oils synergize and antagonize toxicity of diferent conventional insecticides against Myzus persicae (Hemiptera: Aphididae). PLoS ONE 2015, 10, e0127774. [CrossRef]

32. Benelli, G.; Pavela, R.; Iannarelli, R.; Petrelli, R.; Cappellacci, L.; Cianfaglione, K.; Afshar, F.H.; Nicoletti, M.; Canale, A.; Maggi, F. Synergized mixtures of Apiaceae essential oils and related plant-borne compounds: Larvicidal effectiveness on the flariasis vector Culex quinquefasciatus Say. Ind. Crop. Prod. 2017, 96, 186-195. [CrossRef]

33. Mantzoukas, S.; Milonas, P.; Kontodimas, D.; Angelopoulos, K. Interaction between the entomopathogenic bacterium Bacillus thuringiensis subsp. kurstaki and two entomopathogenic fungi in bio-control of Sesamia nonagrioides (Lefebvre) (Lepidoptera: Noctuidae). Ann. Microbiol. 2013, 63, 1083-1091. [CrossRef]

34. Mantzoukas, S.; Zikou, A.; Triantafillou, V.; Lagogiannis, I.; Eliopoulos, P.A. Interactions between Beauveria bassiana and Isaria fumosorosea and their hosts Sitophilus granarius (L.) and Sitophilus oryzae (L.) (Coleoptera: Curculionidae). Insects 2019, 10, 362. [CrossRef] [PubMed]

35. Mantzoukas, S. The effect of Metarhizium robertsii and Bacillus thuringiensis against Helicoverpa armigera (Hübner) (Lepidoptera: Noctuidae). Adv. Ecol. Environ. Res. 2019, 136-146. Available online: https://www.ss-pub.org/aeer/the-effect-of-metarhizium-robertsii-and-bacillus-thuringiensis-againsthelicoverpa-armigera-hubner-lepidoptera-noctuidae/ (accessed on 12 September 2020).

36. Shapiro-Ilan, D.I.; Cottrell, T.E.; Bock, C.; Mai, K.; Boykin, D.; Wells, L.; Hudson, W.G.; Mizell, R.F., 3rd. Control of pecan weevil with microbial biopesticides. Environ. Entomol. 2017, 46, 1299-1304. [CrossRef]

37. Peng, D.; Luo, K.; Jiang, H.; Deng, Y.; Bai1, L.; Zhou, X. Combined use of Bacillus subtilis strain B-001 and bactericide for the control of tomato bacterial wilt. Pest Manag. Sci. 2017, 73, 1253-1257. [CrossRef] [PubMed]

38. Dakhel, W.H.; Latchininsky, A.V.; Jaronski, S.T. Efficacy of two entomopathogenic fungi, Metarhizium brunneum, strain F52 alone and combined with Paranosema locustae against the migratory grasshopper, Melanoplus sanguinipes, under laboratory and greenhouse conditions. Insects 2019, 10, 94. [CrossRef] [PubMed]

39. Xu, X.M.; Jeffries, P.; Pautasso, M.; Jeger, M.J. Combined use of biocontrol agents to manage plant diseases in theory and practice. Phytopathology 2011, 101, 1024-1031. [CrossRef] 
40. Xu, X.M.; Jeger, M.J. Theoretical modeling suggests that synergy may result from combined use of two biocontrol agents for controlling foliar pathogens under spatial heterogeneous conditions. Phytopathology 2013, 103, 768-775. [CrossRef]

41. Portman, S.L.; Krishnankutty, S.M.; Reddy, G.V.P. Entomopathogenic nematodes combined with adjuvants presents a new potential biological control method for managing the wheat stem sawfly, cephus cinctus (Hymenoptera: Cephidae). PLoS ONE 2016, 11, e0169022. [CrossRef]

42. Nathan, S.S.; Kalaivani, K.; Murugan, K. Effect of biopesticides on the lactate dehydrogenase (LDH) of the rice leaffolder, Cnaphalocrocis medinalis (Guenée) (Insecta: Lepidoptera: Pyralidae). Ecotoxicol. Environ. Saf. 2006, 65, 102-107. [CrossRef] [PubMed]

43. Hancock, P.A. Combining fungal biopesticides and insecticide-treated bednets to enhance malaria control. PLoS Comput. Biol. 2009, 5, e1000525. [CrossRef] [PubMed]

44. Köhl, J.; Kolnaar, R.; Ravensberg, W.J. Mode of action of microbial biological control agents against plant diseases: Relevance beyond efficacy. Front. Plant Sci. 2019, 10, 1-19. [CrossRef] [PubMed]

45. Ikbal, C.; Pavela, R. Essential oils as active ingredients of botanical insecticides against aphids. J. Pest Sci. 2019, 92, 971-986. [CrossRef]

46. Lacey, L.A.; Grzywacz, D.; Shapiro-Ilan, D.I.; Frutos, R.; Brownbridge, M.; Goettel, M.S. Insect pathogens as biological control agents: Back to the future. J. Invertebr. Pathol. 2015, 132, 1-41. [CrossRef] [PubMed]

47. Rahim, M. Biological activity of azadirachtin-enriched neem kernel extracts against Rhyzopertha dominica (F.) (Coleoptera: Bostrichidae) in stored wheat. J. Stored Prod. Res. 1998, 34, 123-128. [CrossRef]

48. Tofel, H.K.; Nukenine, E.N.; Stahler, M.; Adler, C. Degradation of azadirachtin A on treated maize and cowpea and the persistence of Azadirachta indica seed oil on Callosobruchus maculatus and Sitophilus zeamais. J. Stored Prod. Res. 2016, 69, 207-212. [CrossRef]

49. Kavallieratos, N.G.; Athanassiou, C.G.; Saitanis, C.J.; Kontodimas, D.C.; Roussos, A.N.; Tsoutsa, M.S.; Anastassopoulou, U. Effect of two azadirachtin formulations against adults of Sitophilus oryzae and tribolium confusum on different grain commodities. J. Food Prot. 2007, 70, 1627-1632. [CrossRef]

50. Robertson, J.L.; Preisler, H.K. Pesticide Bioassays with Arthropods; CRC: Boca Raton, FL, USA, 1992.

51. Xie, Y.S.; Fields, P.G.; Isman, M.B. Repellency and toxicity of azadirachtin and neem concentrates to three stored-product beetles. J. Econ. Entomol. 1995, 88, 1024-1031. [CrossRef]

52. Liu, X.; Zhang, Q.; Xu, B.; Li, J. Effects of Cry1Ac toxin of Bacillus thuringiensis and nuclear polyhedrosis virus of Helicoverpa armigera (Hübner) (Lepidoptera: Noctuidae) on larval mortality and pupation. Pest Manag. Sci. 2006, 62, 729-737. [CrossRef]

53. Moscardi, F. Assessment if the application of baculoviruses for the control of Lepidoptera. Annu. Rev. Entomol. 1999, 44, 257-289. [CrossRef] [PubMed]

54. Hernández, A.F.; Gil, F.; Lacasaña, M. Toxicological interactions of pesticide mixtures: An update. Arch. Toxicol. 2017, 91, 3211-3223. [CrossRef] [PubMed]

55. Espinel-Correal, C.; López-Ferber, M.; Zeddam, J.-L.; Villamizar, L.; Gómez, J.; Cotes, A.M.; Léry, X. Experimental mixtures of Phthorimaea operculella granulovirus isolates provide high biological efficacy on both Phthorimaea operculella and Tecia solanivora (Lepidoptera: Gelechiidae). J. Invertebr. Pathol. 2012, 110, 375-381. [CrossRef] [PubMed]

56. Bhuiyan, K.R.; Hassan, E.; Isman, M.B. Growth inhibitory and lethal effects of some botanical insecticides and potential synergy by dillapiole in Spodoptera litura (Fab.) (Lepidoptera: Noctuidae). J. Plant Dis. Prot. 2000, 108, 82-88.

57. Khorrami, F.; Valizadegan, O.; Forouzan, M.; Soleymanzade, A. The antagonistic/synergistic effects of some medicinal plant essential oils, extracts and powders combined with Diatomaceous earth on the flour beetle, Tribolium castaneum Herbst (Coleoptera: Tnenebrionidae). Arch. Phytopathol. Plant Prot. 2018, 51, 13-14. [CrossRef]

(C) 2020 by the authors. Licensee MDPI, Basel, Switzerland. This article is an open access article distributed under the terms and conditions of the Creative Commons Attribution (CC BY) license (http://creativecommons.org/licenses/by/4.0/). 